\section{GW23-e1020 A PROSPECTIVE STUDY ABOUT THE CARDIAC PERFORMANCE IN THE CHINESE PATIENTS WITH SEPTIC SHOCK: THE SYSTOLIC FUNCTION ASSESSED BY TISSUE DOPPLER IMAGING AND THE BIOMARKERS}

doi:10.1136/heartjnl-2012-302920w.6

Liu Yongtai, Weng Li, Du Bin, Zhang Shuyang. Department of Cardiology, Peking Union Medical College Hospital, Peking Union Medical College and Chinese Academy of Medical Sciences, Beijing, China

Objectives This study was to analyse evolution of the left ventricular (LV) systolic function assessed by Tissue Doppler imaging (TDI) and its predictive value to the prognosis in Chinese patients with septic shock, and to evaluate the correlation between these parameters and cardiac biomarkers including cardiac tropnin I (cTnI) and N-terminal-pro-BNP (NT-proBNP).

Methods This study prospectively recruited 69 patients within $24 \mathrm{~h}$ after the onset of septic shock. The clinical variables, cardiac biomarkers and echocardiography data were obtained and analysed on admission (day 1) and at day 2, 4, 7 and 10 or $24 \mathrm{~h}$ after the correction of shock. The primary endpoint was 90 days all-cause mortality.

Results The 90 days all-cause mortality which was $40.6 \%$ in this study. The survivors had a lower peak myocardial systolic velocity $(\mathrm{Sm})$ which emerged as an independent predictor of the 90 days mortality in the multivariate analysis at day 1 . There was no difference in the baseline level of biomarkers. During the mean follow-up period of 4.8 days, NT-proBNP and cTnI decreased significantly and Sm improved significantly in the survivors; the non-survivors showed a significantly increased NT-proBNP, unchanged cTnI and Sm. The biomarkers correlated significantly with Sm and LVEF both in the baseline and in the follow-up only in the survivors. The correlation of NTproBNP with cTnI remained significant throughout the follow-up period in both groups.

Conclusions LV systolic function assessed by TDI related to the prognosis of patients with septic shock. The level of biomarkers decreased and strong correlations could be found between the biomarkers and LV systolic function in the 90 days survivors after the onset of septic shock.
${ }^{1}$ Zhang Jun, ${ }^{2}$ Zhou Bin, ${ }^{1}$ Wang Cheng, 'Li Cuicui, ${ }^{1}$ Zhao Wenbo, ${ }^{1}$ Li Ming, 'Lou Tanqi.

${ }^{1}$ Department of Nephrology, Third Affiliated Hospital, Sun Yat-sen University;

${ }^{2}$ Department of Cardiology, Third Affiliated Hospital, Sun Yat-sen University

Objectives Previous studies have shown that depressive symptoms are associated with poor cardiovascular outcomes in patients with chronic kidney disease (CKD). However, the association between depressive symptoms and 24-h blood pressure (BP) patterns in this population is unclear. In the current study, we aimed determine the association between the non-dipping status and depression in patients with CKD in a cross-sectional study.

Methods A total of 221 patients with CKD were recruited into this study. Sleep quality was measured by Pittsburgh Sleep Quality Index (PSOI), while depressive symptoms were assessed by Beck Depression Inventory-13 item (BDI-13). 24-h BP patterns were determined by 24-h ambulatory BP monitoring. eGFR were assessed by simplified MDRD equation.

Results A total of 93 (42.9\%) and 128 (57.1\%) patients were defined as dippers and non-dippers, respectively. Non-dippers had higher daytime mean systolic BP and diastolic BP (135.3 \pm 18.8 vs 128.1 $\pm 16.7, p=0.002 ; 80.9 \pm 11.3$ vs $77.9 \pm 10.7, p=0.046$ ), higher nocturnal systolic BP and nocturnal diastolic BPs $(135.3 \pm 20.1$ vs 117.1 $\pm 15.7, p=0.000 ; 80.9 \pm 12.3$ vs $71.2 \pm 10.7, p=0.000)$, In univariate analyses, non-dippers had higher BDI scores $(6.27 \pm 5.42$ vs $4.41 \pm 4.10, \mathrm{p}=0.007)$ and higher PSOI scores $(9.89 \pm 4.87$ vs 8.60 $\pm 4.40, \quad p=0.037)$, older age $(41.76 \pm 15.38$ years vs 35.71 \pm 13.12 years, $p=0.001)$ than dippers. In addition, non-dippers had had lower eGFR level than dippers $(55.7 \pm 47.8$ vs $77.8 \pm 47.1$, $\mathrm{p}=0.001)$. Multiple logistic regression analyses showed non-dipping status was associated with high $\mathrm{BDI}$ scores $(\mathrm{OR}=1.07,95 \% \mathrm{CI}$ 1.03 to 1.12 ), eGFR (OR $=0.98,95 \%$ CI 0.96 to 0.99 ) and ambulatory systolic blood mean pressure ( $\mathrm{OR}=1.12,95 \% \mathrm{CI} 1.03$ to 1.42 ). In this model, there were no significant associations between nondipping and age, sex, ambulatory diastolic blood mean pressure, PSOI score.

Conclusions Our study showed a high prevalence of non-dipping blood pressure in CKD patients. In view of the adverse effects of non-dipping on outcomes of patients with CKD, improvement of depressive symptoms might benefit this population. 